\title{
Coupled Processes of Fluid Flow, Solute Transport, and Geochemical Reactions in Reactive Barriers
}

Jeongkon Kim*, Franklin W. Schwartz, and Tianfu Xu, Heechul Choi, and In S. Kim

Jeongkon Kim, Korea Institute of Water and Environment, Korea Water Resources Corporation, Daejon, Korea;

Franklin W. Schwartz, Department of Geological Sciences, The Ohio State University, Columbus, OH 43210;

Tianfu Xu, Earth Sciences Division, Lawrence Berkeley, National Laboratory, Berkeley CA, 94720; Heechul Choi

and In S. Kim, Department of Environmental Science and Engineering, Kwangju Institute of Science and

Technology, Gwangju, Korea. *Corresponding author (jkkim@kowaco.or.kr). 


\begin{abstract}
A complex pattern of coupling between fluid flow and mass transport develops when heterogeneous reactions occur. For instance, dissolution and precipitation reactions can change the physical properties of a medium, such as permeability and pore geometry. These changes influence fluid flow, which in turn impact the composition of dissolved constituents and solidphase, and the rate and direction of advective transport. Two-dimensional modeling studies using TOUGHREACT were conducted to investigate the coupling between flow and transport developed as a consequence of difference in density, dissolution/precipitation, and medium heterogeneity. The model includes equilibrium reactions for aqueous species, kinetic reactions between the solid phases and aqueous constituents, and full coupling of porosity and permeability changes resulting from precipitation and dissolution reactions in porous media. In addition, a new permeability relationship is implemented in TOUGHREACT to examine the effects of geochemical reactions and density difference on plume migration in porous media. Generally, the evolutions in the concentrations of the aqueous phase are intimately related to the reaction-front dynamics. Plugging of the medium contributed to significant transients in patterns of flow and mass transport.
\end{abstract}




\section{INTRODUCTION}

Often, waters from landfills or mining operations contain elevated concentrations iron and other metals at low $\mathrm{pH}$. A promising approach to minimize groundwater contamination by acidic metallic compounds is the use of calcareous reactive barriers. For example, in a ferriccalcareous barrier, hydrolysis of $\mathrm{Fe}^{3+}$ generates $\mathrm{H}^{+}$, which is neutralized by reaction with calcite, causing the formation of poorly crystalline ferric oxyhydroxide. Because iron oxyhydroxides form hydrous aggregates capable of reducing porosity and permeability, their precipitation along the front of a contaminant plume is potentially useful in slowing the spread of the contaminant (Fryar and Schwartz, 1994). The essence of this technology is the combination of containment, in which low-permeability barriers are installed around the contaminant plume, with in situ treatment, in which reactions are induced to immobilize contaminants.

The main objective of this study is to evaluate the impact of dissolution/precipitation and heterogeneity on mass transport and reaction zone development in a two-dimensional reactive barrier system.

\section{PROBLEM DESCRIPTION}

In this study, chemical evolution within a ferric-calcareous barrier is examined using a two-dimensional model. Specifically, we demonstrate the coupled effects of variable density fluid flow and heterogeneous reactive mass transport and their influence on the reaction front development. The aqueous species involved in the reaction are $\mathrm{H}^{+}, \mathrm{OH}^{-}, \mathrm{Cl}^{-}, \mathrm{Na}^{+}, \mathrm{Fe}^{3+}, \mathrm{ClO}_{4}^{-}$, $\mathrm{Ca}^{2+}, \mathrm{H}_{2} \mathrm{CO}_{3}^{0}, \mathrm{HCO}_{3}^{-}$and $\mathrm{CO}_{3}^{-}$, and the mineral phases are calcite and $\mathrm{Fe}(\mathrm{OH})_{3}(\mathrm{~s})$. 
Shi (2000) conducted a series of experiments using a flow tank measuring $1.82 \mathrm{~m}$ long, $0.62 \mathrm{~m}$ high, and $0.10 \mathrm{~m}$ deep, providing a total volume and inflow area of $0.1133 \mathrm{~m}^{3}$ and $0.062 \mathrm{~m}^{2}$, respectively. The tank was evenly filled with coarse-grained glass beads $(0.75 \mathrm{~mm})$ mixed with $0.3 \mathrm{wt} \%$ calcite. The inflow rate was fixed at a linear flow velocity of $2.56 \mathrm{~cm} / \mathrm{hr}$. An acidic $(\mathrm{pH}$ 2.5) contaminant solution consisting of $2000 \mathrm{mg} / \mathrm{l} \mathrm{NaCl}$ (conservative tracer) and $300 \mathrm{mg} / \mathrm{l}$ $\mathrm{Fe}\left(\mathrm{ClO}_{4}\right)_{3}$ (reactive tracer) was injected at $65.0 \mathrm{ml} / \mathrm{min}$ at an injection point $(\mathrm{x}=0.2 \mathrm{~m}$ and $\mathrm{z}=$ $0.2 \mathrm{~m})$. The experiments were conducted at about $20^{\circ} \mathrm{C}$.

The numerical simulations were conducted employing the same conditions used in the experiment. A computational mesh was created for the model domain using 3,552 elements (74 columns x 48 rows). Modeling parameters are summarized in Table 1. Assuming that the effects of calcite on the porosity and hydraulic conductivity are negligible, the hydraulic conductivity values for the $0.75 \mathrm{~mm}$ glass beads are estimated to be $4.20 \times 10^{-2} \mathrm{~cm} / \mathrm{sec}$. Total concentrations for the resident, inlet, and injection fluids are listed in Table 2. The boundaries at the top and bottom of the tank are impermeable. The elements of the right boundary for flow are assigned constant pressures estimated at hydrostatic condition, while the elements on the left boundary for flow are assigned either a constant flux or constant pressures depending on specific problems. Boundary conditions for concentrations are assigned as a Dirichlet boundary at the left side of the tank, and a free-exit boundary at the right. All other concentration boundaries are assigned as no-flux. Initial dissolved concentrations are assigned values that are in equilibrium with calcite throughout the domain. The fluids of the tank, inlet, and injection source were estimated to be $998.2,998.2$, and $999.7 \mathrm{~kg} / \mathrm{m}^{3}$, respectively, at $20^{\circ} \mathrm{C}$. The concentrations of the inlet fluid are different from those of the resident fluid because the dissolved species only in the resident solution are in equilibrium with calcite. 


\section{Model Description}

The simulations are carried out using the non-isothermal reactive geochemical transport code TOUGHREACT (Xu and Pruess, 1998). This code was developed by introducing reactive chemistry into the framework of the existing multi-phase fluid and heat flow code TOUGH2 (Pruess, 1991). The flow and transport in geologic media are based on space discretization by means of integral finite differences (Narasimhan and Witherspoon, 1976). An implicit timeweighting scheme is used for the individual components of the model consisting of flow, transport, and geochemical reaction. TOUGHREACT uses a sequential iteration approach, which solves the transport and the reaction equations separately. The system of chemical reaction equations is solved by Newton-Raphson iteration. Full details on the numerical methods are given in Xu and Pruess (1998). The model can accommodate any number of chemical species present in liquid, gas and solid phases. Local equilibrium is assumed to govern the distribution of aqueous chemical species. Mineral dissolution/ precipitation can proceed subject either to local equilibrium or to kinetic conditions.

\section{Permeability-Porosity Relationship}

TOUGHREACT fully accounts for porosity and permeability changes that occur as a result of chemical reactions in porous media by employing the Carman-Kozeny model (Bear, 1972), a macroscopic model that considers the porous media as a bundle of cylindrical pores. However, the conditions we deal with are far more complicated in many aspects as:

(1) Calcite grains mixed with glass beads are likely to exist unconnected in the porous medium given the small mass fraction used in this study $(0.3 \mathrm{wt} \%)$. Thus, dissolution of calcite grains is unlikely directly related to the permeability change. 
(2) Iron commonly exists in two oxidation states: Fe(II) and $\mathrm{Fe}(\mathrm{III})$. Fe(III) in a spent acid will precipitate as gelatinous ferric hydroxide when the $\mathrm{pH}$ of the acid rises above 2 (Crowe, 1986), which will result in plugging when only small quantities of solids have precipitated.

(3) $\mathrm{CO}_{2}$ gas exsolution due to the dissolution of calcite was found to reduce hydraulic conductivity by 2 or more orders of magnitude, causing the plume to decelerate (Fryar and Schwartz, 1998).

There is no information available on the effects of calcite grains existing unconnected on changes in permeability and hydraulic conductivity. In addition, the current version of TOUGHREACT does not account for direct coupling of gas exsolution and phase change in density dependent flow module. The porous media used in this study is very uniform with simple geometry (e.g., $0.75 \mathrm{~mm}$ glass beads), which excludes the use of microscopic models based on a "cut-and-random-rejoin" approach assuming that pores of different radii are randomly distributed within the porous media and hydraulic conductivity is determined by the inter-connectivity of the pores.

Accordingly, a new permeability-porosity relationship was developed and implemented in TOUGHREACT to approximate changes in permeability and hydraulic conductivity by employing lumped parameters as a function of $\mathrm{Fe}(\mathrm{OH})_{3}(\mathrm{~s})$. It is assumed that the contribution of calcite dissolution to permeability change is negligible under the conditions of this study. The new permeability-porosity function has the following form:

$$
k=k_{i} \exp \left(-\alpha\left(\frac{\phi_{\mathrm{Fe}(\mathrm{OH})_{3}}}{\phi_{i}}\right)^{\beta}\right)
$$


where $k$ is the permeability; $k_{i}$ is the intrinsic permeability; $\phi_{i}$ and $\phi_{\mathrm{Fe}(\mathrm{OH})_{3}}$ are the initial porosity

and the volume fraction of $\mathrm{Fe}(\mathrm{OH})_{3}(\mathrm{~s})$; and $\alpha$ and $\beta$ are fitting constants. Taylor and Jaffé (1990)

used a similar function to describe permeability reduction due to biomass growth in porous media.

\section{RESULTS AND DISCUSSION}

\section{Permeability-Porosity Relationship Comparison}

Simulation results obtained using the simplified Carman-Kozeny model, as implemented in TOUGHREACT are compared with one of many trial simulation results conducted using equation (1) to determine a set of $\alpha$ and $\beta$ that reasonably describes development of reaction zone and dissolved plumes of experimental data of Shi (2000).

Figure 1 shows the experimental results obtained at 38 day under the same condition as with the numerical simulations. Figures 2(a) - (e) show profiles for permeability, $\mathrm{Fe}(\mathrm{OH})_{3}(\mathrm{~s}), \mathrm{Fe}^{3+}, \mathrm{pH}$, and $\mathrm{Cl}^{-}$, respectively, obtained using the Carman-Kozeny equation. Permeability of the porous media increased in most of the reaction zones (Figure 2a), which indicates that calcite dissolution has a greater impact on permeability change than $\mathrm{Fe}(\mathrm{OH})_{3}(\mathrm{~s})$ precipitation. As a result, all profiles show a thin, elongate plume shape (Figures $2 \mathrm{~b}-2 \mathrm{e}$ ). The advective flow carries the solute plume laterally across the domain with much vertical spreading because of the lack of permeability reduction.

Depicted in Figures 3(a) - (e) are results of the same simulations using the new permeability-porosity relationship with $\alpha=50.0$ and $\beta=0.5$. This set of alpha and beta values is found to best describe experimental data. Unlike the profiles in Figure 2, profiles for permeability, $\mathrm{Fe}(\mathrm{OH})_{3}(\mathrm{~s}), \mathrm{Fe}^{3+}, \mathrm{pH}$, and $\mathrm{Cl}^{-}$in Figure 2 indicate a significant vertical spreading resulting from permeability reduction in the reaction front as the acid plume migrates. In both 
cases, there are similar degrees of precipitation and dissolution as shown in Figures $1 \mathrm{~b}$ and $2 \mathrm{~b}$. Precipitation in Figure 1, however, occurs along a narrow, long strip because of the lack of permeability reduction calculated using the Carman-Kozeny model. However, in Figure 3 permeability reduction as calculated using Equation (1) is much greater, resulting in a wide, short precipitation zone. The effect of density on vertical plume migration is more obvious in Figure 2 than in Figure 2 because of reduced horizontal flow velocity caused by stronger permeability reduction in Figure 3.

\section{Reaction Zone Development}

Figure 4 presents the evolution of $\mathrm{Fe}(\mathrm{OH})_{3}(\mathrm{~s})$ precipitation and re-dissolution dynamics as the heterogeneous reaction taking place between different phases (i.e., solid and liquid phases) progresses. The development of the reaction zones is a continuous process in which the heterogeneous reactions (dissolution and precipitation) evolve sequentially or simultaneously as calcite comes into contact with the introduced contaminants. One of primary concerns in a reactive barrier, such as a calcareous wall is the effective hydraulic behavior of the reactive barrier. Figure 3 clearly shows that a complex pattern of coupling between fluid flow and mass transport develops when heterogeneous reactions occur.

The coupling between geochemical reactions caused by the injection of $\mathrm{Fe}\left(\mathrm{ClO}_{4}\right)_{3}$ solution and the density-dependent flow influence the reactions and dissolved plume migration in a complicated manner. Although the main migration direction of the dissolved plume is controlled by the ambient flow of water, density-driven flow related to the large concentration of dissolved ions in the injected source water caused significant vertical movement to develop in both the dissolved plume and the reaction zone. In this study, there is approximately $0.15 \%$ density 
difference between the ambient and injection waters, based on the density of water estimated at $2000 \mathrm{mg} / \mathrm{L} \mathrm{NaCl}$ at $20^{\circ} \mathrm{C}$. Note that the current version of TOUGHREACT could not account for multi-species in calculating density changes. Thus, it was assumed that solute density could be calculated based on $\mathrm{NaCl}$ concentration only by neglecting contributions from other species, which can be justified by the fact that $\mathrm{NaCl}$ concentration was much greater than other species.

The reaction zones propagated much more slowly than the dissolved plume. While the non reactive species $\left(\mathrm{Cl}^{-}, \mathrm{Na}^{+}, \mathrm{ClO}^{-}, \mathrm{Ca}^{2+}\right)$ spread beyond the reactions zone (Figure 3), the

reactive species $\left(\mathrm{Fe}^{3+}\right)$ is effectively retained in the reaction zone and moves much slowly (Figure 4). The final shape of the reaction zone was significantly influence by the geochemical reactions in addition to the density gradients.

\section{Effect of Heterogeneity}

Heterogeneities influence the pattern of reactions in a highly complex and nonlinear manner. Heterogeneity in hydraulic conductivity is another factor that influences the pathway of reactive plumes. For instance, local heterogeneity can perturb the interface and promote the formation of fingering in dense plumes (Schincariol, 1998).

Previous simulations discussed here assume that the porous medium is both chemically (calcite content) and physically (permeability) homogeneous. However, it is inevitable that some sort of heterogeneity is created even under well designed experimental conditions. In this section, simulation results from chemically and physically heterogeneous porous media are presented.

Spatially variable random fields for calcite content and for permeability are generated using the Fourier spectral technique of Robin et al. (1993) with correlation lengths of $2.54 \mathrm{~cm}$ and $1.27 \mathrm{~cm}$ in the $\mathrm{x}$ - and $\mathrm{z}$-directions, respectively. Figures 5(a) and (b) show the random fields 
for calcite content and permeability, respectively. The mean and variance of the calcite content random field are $0.302 \mathrm{wt} \%$ and 0.088 , respectively, and those of the permeability (ln k) are 21.55 and 0.695 , respectively. Note, however, that as the correlation lengths of $2.54 \mathrm{~cm}$ and 1.27 $\mathrm{cm}$ are identical to the grid resolution, the spatial correlation as described by a variogram is not really resolved.

Figures 6 and 7 shows the profiles for permeability, $\mathrm{Fe}(\mathrm{OH})_{3}(\mathrm{~s}), \mathrm{Fe}^{3+}, \mathrm{pH}$, and $\mathrm{Cl}^{-}$for the chemical heterogeneous case and for the physical heterogeneous case, respectively. It is clear that both chemical and physical heterogeneities significantly impact the progress of heterogeneous reactions and dissolved plumes in the calcite reactive barrier.

As shown in Figures 6 and 7, both physical and chemical heterogeneities are key factors affecting permeability changes in response to precipitation along the fast flowing paths. Averaging within a grid block (and also in a homogeneous system) must have greatly reduced our ability to capture clogging effects in detail. That is, the results may change, depending on the grid size. For example, permeability reductions might be less evident if larger grid sizes was used due to the averaging effect.

\section{CONCLUSIONS}

We have examined coupled and geochemically reactive systems in homogeneous and heterogeneous porous media using TOUGHREACT. A new permeability-porosity relationship model was developed and implemented in TOUGHREACT. We demonstrate the coupled effects of variable density fluid flow and heterogeneous reactive mass transport and their influence on developing reaction front. Generally, the evolutions in the concentrations of the aqueous phase are clearly related to the reaction-front dynamics. Plugging of the medium contributed to significant transients in patterns of flow and mass transport. Although the new permeability 
model describes approximately the general trend of reactive plume migration under heterogeneous reactions, this approach is subject to further verification.

\section{ACKNOWLEDGMENT}

Funding for this study was provided by the National Science Foundation, EAR-9814820. This research was also supported by a grant (4-1-1) from Sustainable Water Resources Research Center (SWRRC) of 21st century frontier R\&D program through the Water Reuse Technology Center (WRTC) at Kwangju Institute of Science \& Technology (K-JIST).

\section{REFERENCES}

Bear, J. 1972. Dynamics of fluids in porous media, American Elsevier, New York, NY.

Crowe, C.W. 1986. Precipitation of hydrated silica from spent hydrofluoric acid: how much of a problem is it?, J. Pet. Technol., pp. 1234-1240.

Freeze, R.A., Cherry, J.A. 1979. Groundwater, Prentice-Hall, Englewood Cliffs, N. J.

Fryar, A.E., Schwartz, F.W. 1998. Hydraulic-conductivity reduction, reaction-front propagation, and preferential flow within a model reactive barrier. J. Contam. Hydr, 32(3-4), 333351.

Fryar, A.E., Schwartz, F.W. 1994. Modeling the removal of metals from ground water by a reactive barrier: Experimental results. Water Resour. Res., 30(12), 3455-3469.

Narasimhan, T.N., Witherspoon, P.A. 1976. An integrated finite difference method for analyzing fluid flow in porous media, Water Resour. Res. 12, 57-64. 
Pruess, K. 1991. TOUGH2: A general numerical simulator for multiphase fluid and heat flow, Lawrence Berkeley Laboratory Report LBL-29400, Berkeley, California.

Raiders, R.A., McInerney, M.j., Revus, D.E., Torbati, H.M., Knapp, R.M., Jenneman, G.E. 1986. Selectivity and depth of microbial plugging in Berea sandstone cores, J. Ind. Microbiol., 1, 195-203.

Robin, M.J.L., Gutjahr, A.L., Sudicky, E.A., Wilson, J.L. 1993. Cross-correlated random field generation with the direct Fourier transform method, Water Resour. Res., 29(7), 23852397.

Schincariol, R.A. 1998. Dispersive mixing dynamics of dense miscible plumes: natural perturbation initiation by local-scale heterogeneities. J. Contam. Hydr., 34(3), 247-271.

Shi, J. 2000. Conservative and reactive mass transport in homogenous and heterogeneous systems, Dissertation, The Ohio State University, Columbus, Ohio.

Taylor, S.W., Jaffé, P.R. 1990. Biofilm growth and the related changes in the physical properties of a porous medium, 1, Experimental investigation, Water Resour. Res., 26(9), 215321590.

Xu, T., Pruess, K. 1998. Coupled modeling of non-isothermal multiphase flow, solute transport and reactive chemistry in porous and fractured media: 1. Model development and validation. Lawrence Berkeley National Laboratory Report LBNL-42050, Berkeley, California, 38 pp. 


\section{FIGURE CAPTIONS}

Figure 1. Reaction zone (red) and total Fe concentrations (mg/l) in pore water (Contour lines) observed at 38 days (Shi, 2000).

Figure 2. Profiles using the Cozeny-Karman model at 38 days for (a) permeability, (b) $\mathrm{Fe}(\mathrm{OH})_{3}(\mathrm{~s}),(\mathrm{c}) \mathrm{Fe}^{3+}$, (d) $\mathrm{pH}$, and (e) $\mathrm{Cl}^{-}$.

Figure 4. Profiles using equation (1) at 38 days for (a) permeability, (b) $\mathrm{Fe}(\mathrm{OH})_{3}(\mathrm{~s})$, (c) $\mathrm{Fe}^{3+}$, (d) $\mathrm{pH}$, and (e) $\mathrm{Cr}^{-}$.

Figure 4. Development of reaction zones.

Figure 5. Random fields for (a) calcite content and (b) permeability.

Figure 6. Profiles for (a) permeability, (b) $\mathrm{Fe}(\mathrm{OH})_{3}(\mathrm{~s})$, (c) $\mathrm{Fe}^{3+}$, (d) $\mathrm{pH}$, and (e) $\mathrm{Cl}^{-}$in the chemically heterogeneous porous medium at 38 days.

Figure 7. Profiles for (a) permeability, (b) $\mathrm{Fe}(\mathrm{OH})_{3}(\mathrm{~s})$, (c) $\mathrm{Fe}^{3+}$, (d) $\mathrm{pH}$, and (e) $\mathrm{Cl}^{-}$in the physically heterogeneous porous medium at 38 days. 
Table 1. Parameter values for numerical simulations.

\begin{tabular}{lc}
\hline Property & Value \\
\hline Porosity & 0.38 \\
Permeability & $4.3 \times 10^{-10} \mathrm{~m}^{2}$ \\
Diffusion coefficient & $1.0 \times 10^{-9} \mathrm{~m}^{2} / \mathrm{s}$ \\
Domain Length & $1.82 \mathrm{~m}$ \\
Domain width & $0.62 \mathrm{~m}$ \\
Horizontal grid size & $2.54 \times 10^{-2} \mathrm{~m}$ \\
Vertical grid size & $1.27 \times 10^{-2} \mathrm{~m}$ \\
\hline
\end{tabular}


Table 2. Total concentrations for the initial, influent, and injection fluids.

\begin{tabular}{lccc}
\hline Aqueous Species & \multicolumn{3}{c}{ Total Concentration (mol/l) } \\
& Initial & Influent & Injection \\
\hline $\mathrm{H}^{+}$ & $5.0 \times 10^{-9}$ & $1.0 \times 10^{-7}$ & $2.0 \times 10^{-3}$ \\
$\mathrm{Na}^{+}$ & $1.0 \times 10^{-7}$ & $1.0 \times 10^{-7}$ & $3.4 \times 10^{-2}$ \\
$\mathrm{Fe}^{3+}$ & $1.0 \times 10^{-7}$ & $1.0 \times 10^{-7}$ & $8.5 \times 10^{-4}$ \\
$\mathrm{Ca}^{2+}$ & $5.0 \times 10^{-4}$ & $3.0 \times 10^{-5}$ & $3.0 \times 10^{-7}$ \\
$\mathrm{Cl}^{-}$ & $1.0 \times 10^{-7}$ & $3.0 \times 10^{-7}$ & $3.4 \times 10^{-2}$ \\
$\mathrm{ClO}_{4}^{-}$ & $3.0 \times 10^{-7}$ & $3.0 \times 10^{-7}$ & $2.5 \times 10^{-3}$ \\
$\mathrm{HCO}_{3}^{-}$ & $1.0 \times 10^{-3}$ & $2.0 \times 10^{-5}$ & $1.0 \times 10^{-5}$ \\
\hline
\end{tabular}




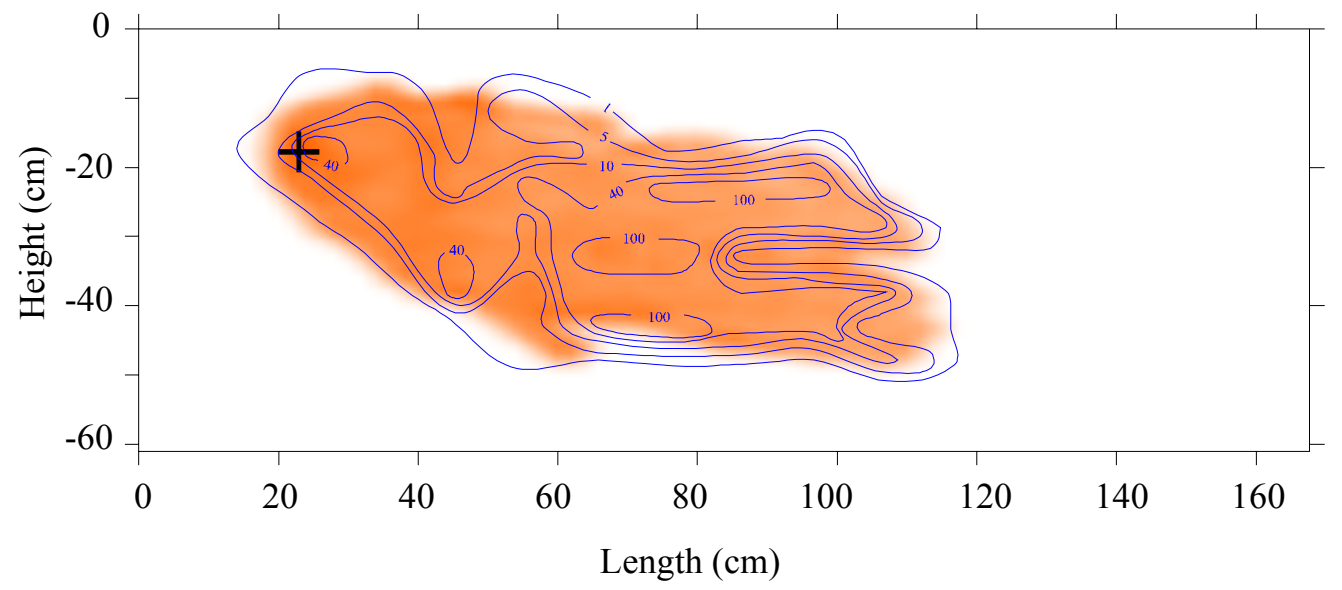

Figure 1. Reaction zone (red) and total Fe concentrations (mg/l) in pore water (Contour lines) observed at 38 days (Shi, 2000). 

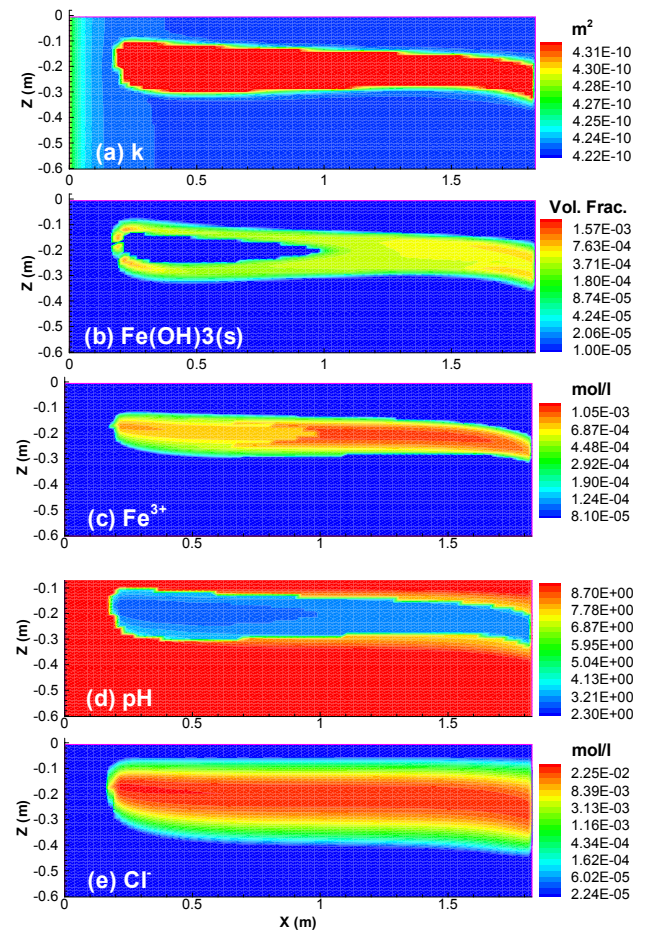

Figure 2. Profiles using the Carman-Kozeny model at 38 days for (a) permeability, (b) $\mathrm{Fe}(\mathrm{OH})_{3}(\mathrm{~s})$, (c) $\mathrm{Fe}^{3+}$, (d) $\mathrm{pH}$, and (e) $\mathrm{Cl}^{-}$. 


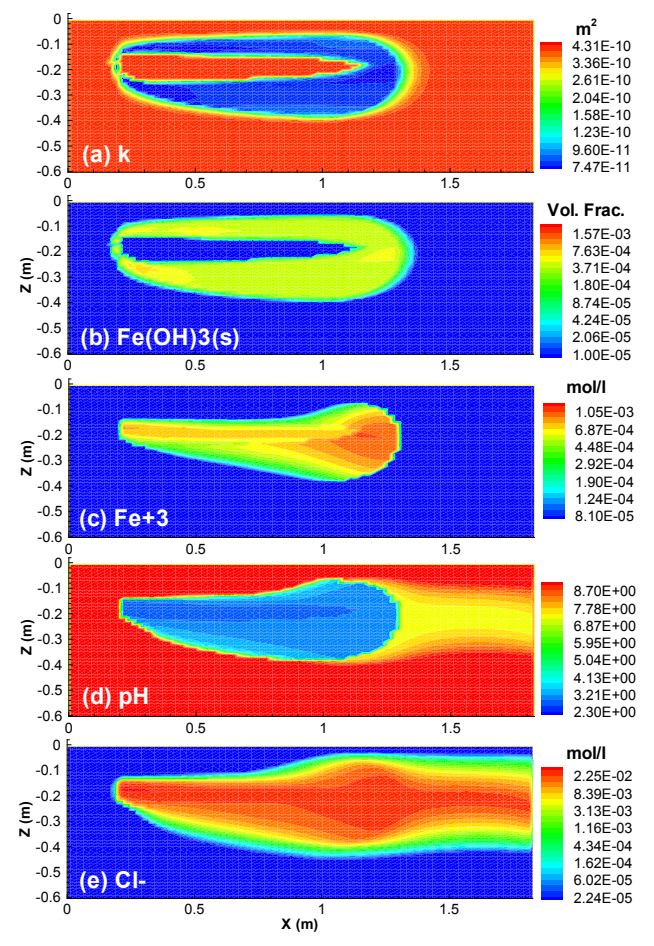

Figure 3. Profiles using equation (1) at 38 days for (a) permeability, (b) $\mathrm{Fe}(\mathrm{OH})_{3}(\mathrm{~s})$, (c) $\mathrm{Fe}^{3+}$, (d) $\mathrm{pH}$, and (e) $\mathrm{Cl}^{-}$. 


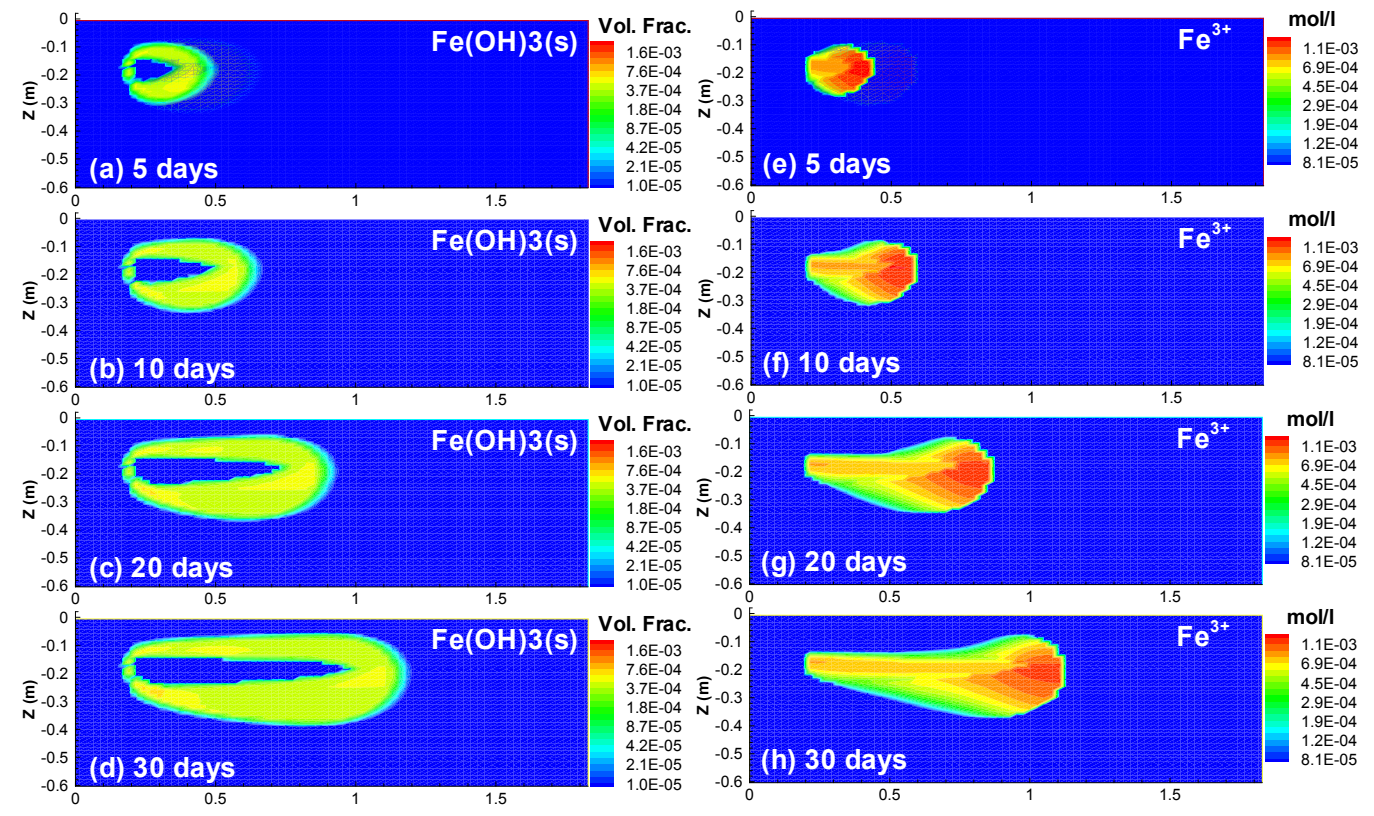

Figure 4. Development of reaction zones. 


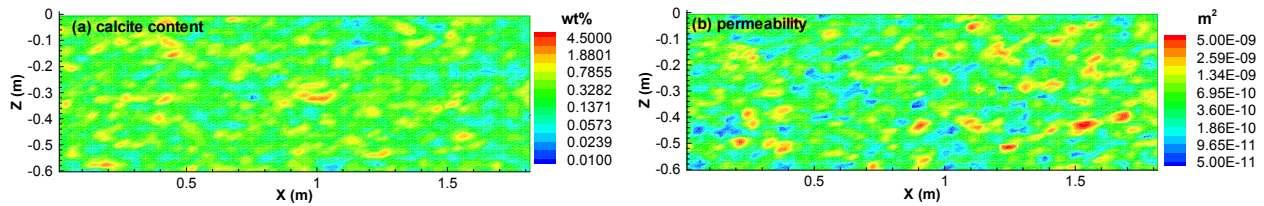

Figure 5. Random fields for (a) calcite content and (b) permeability. 

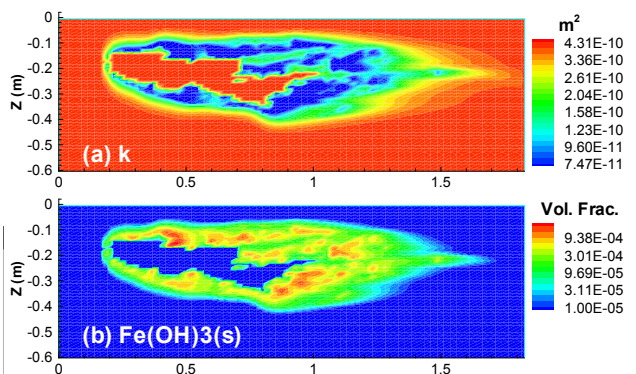

Vol. Frac.
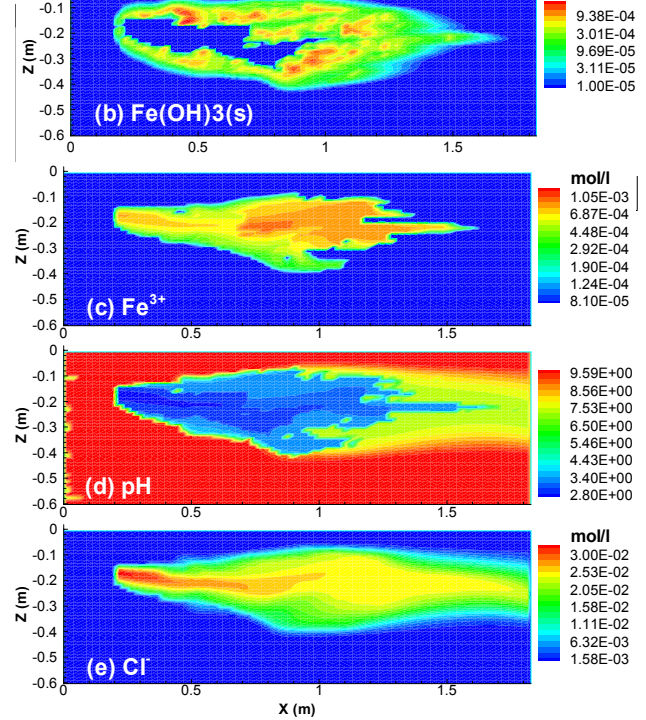

Figure 6. Profiles for (a) permeability, (b) $\mathrm{Fe}(\mathrm{OH})_{3}(\mathrm{~s})$, (c) $\mathrm{Fe}^{3+}$, (d) $\mathrm{pH}$, and (e) $\mathrm{Cl}^{-}$in the chemically heterogeneous porous medium at 38 days. 


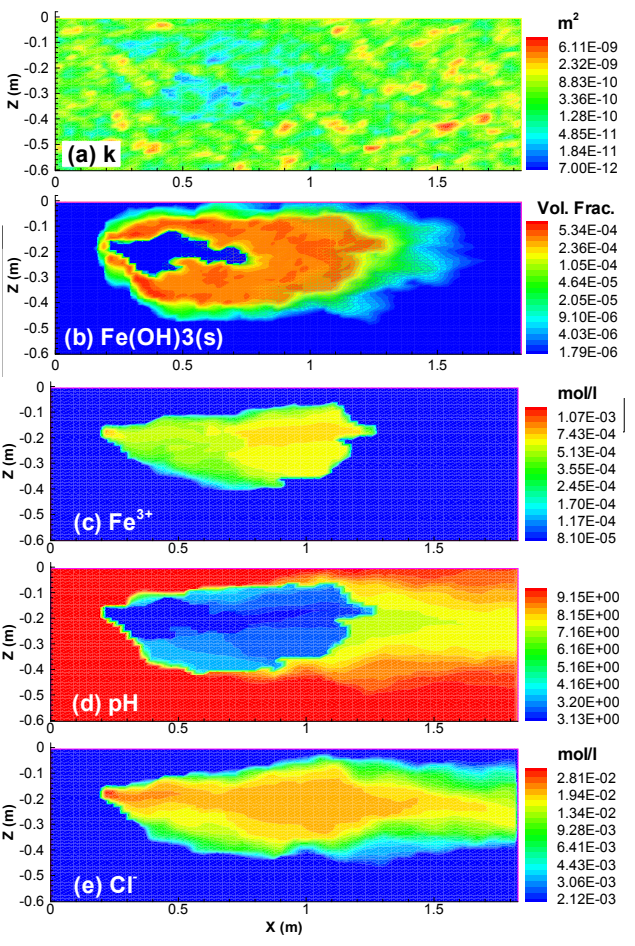

Figure 7. Profiles for (a) permeability, (b) $\mathrm{Fe}(\mathrm{OH})_{3}(\mathrm{~s})$, (c) $\mathrm{Fe}^{3+}$, (d) $\mathrm{pH}$, and (e) $\mathrm{Cl}^{-}$in the physically heterogeneous porous medium at 38 days. 